\section{Estudo de custo-análise do tratamento da artrite reumatoide grave em um município do Sul do Brasil}

\author{
Cost analysis of treatment for severe rheumatoid \\ arthritis in a city in southern Brazil
}

\author{
Estudio analítico de costes del tratamiento por \\ artritis reumatoide severa en una ciudad del \\ sur de Brasil
}

\footnotetext{
${ }^{1}$ Universidade Federal de Santa Catarina, Florianópolis, Brasil. 2 Universidade do Sul de Santa Catarina, Tubarão, Brasil.

${ }^{3}$ Hospital Universitário Professor Polydoro Ernani de São Thiago, Universidade Federal de Santa Catarina, Florianópolis, Brasil.

Correspondência F. B. Buendgens

Universidade Federal de Santa Catarina.

Campus Universitário Reitor João David Ferreira Lima Florianópolis, SC 88040-900, Brasil.

fabiola_bagatini@yahoo. com.br
}

\begin{abstract}
Treatment of rheumatoid arthritis involves the use of medicines, non-pharmaceutical therapies, medical appointments, and complimentary tests, among other procedures. Based on sources of payment, this article presents the direct medical costs related to treatment of rheumatoid arthritis. The cost analysis included 103 patients with severe rheumatoid arthritis treated at the Specialized Division of Pharmaceutical Care in Florianopolis, Santa Catarina State, Brazil. Total annual direct cost was $R \$ 2,045,596.55$ (approximately one million US dollars), or R\$19,860.16 per patient/year (slightly less than ten thousand US dollars). Total cost breakdown was as follows: 90.8\% for medicines, $2.5 \%$ for hospitalizations, $2.2 \%$ for complimentary tests, $2.1 \%$ for medical appointments, and $2.4 \%$ for all other costs. The public sector accounted for $73.6 \%$ of the total direct medical costs and $79.3 \%$ of the cost of medicines. The cost analysis provided a profile of how a group of individuals with a chronic non-communicable disease that requires resources circulates in the public-private mix that characterizes the Brazilian health system.
\end{abstract}

Rheumatoid Arthritis; Health Care Costs; Health Technology Assessment
Fabíola Bagatini Buendgens 1

Carine Raquel Blatt 2

Antônio Carlos Estima Marasciulo ${ }^{3}$

Silvana Nair Leite 1

Mareni Rocha Farias 1

\section{Resumo}

O tratamento da artrite reumatoide envolve a utilização de medicamentos, terapias não farmacológicas, consultas médicas, exames complementares, entre outros procedimentos. O artigo apresenta, conforme as fontes pagadoras, os custos diretos médicos relacionados ao tratamento da artrite reumatoide. Trata-se de um estudo de custo-análise envolvendo 103 pacientes com artrite reumatoide grave atendidos por meio do Componente Especializado da Assistência Farmacêutica em Florianópolis, Santa Catarina, Brasil. O custo direto médico total foi $R \$ 2.045 .596,55 /$ ano, correspondendo a $R \$ 19.860,16$ por pacientelano. Do custo total, 90,8\% foram para despesas com medicamentos, 2,5\% às hospitalizações, 2,2\% aos exames complementares, $2,1 \%$ às consultas médicas e 2,4\% à soma dos demais componentes. O setor público foi responsável por 73,6\% do custo direto médico total e por $79,3 \%$ do custo com a aquisição de medicamentos. A análise dos custos permitiu traçar um perfil de como uma população portadora de doença crônico-degenerativa altamente demandante de recursos transita pela composição público-privada que caracteriza o sistema de saúde brasileiro.

Artrite Reumatoide; Custos de Cuidados de Saúde; Avaliação de Tecnologias de Saúde 


\section{Introdução}

A artrite reumatoide é uma doença inflamatória crônica, de origem autoimune, caracterizada por acometimento poliarticular periférico, simétrico e progressivo que, em alguns casos, pode ocasionar comprometimento extra-articular 1,2. O envolvimento da membrana sinovial, com posterior erosão óssea e destruição articular, frequentemente leva a um quadro grave, que determina deformidades e incapacidade funcional do indivíduo ${ }^{3}$.

Estudos internacionais estimam que 0,5\%$1 \%$ da população mundial é acometida pela artrite reumatoide, sendo que as mulheres são mais afetadas do que os homens 4,5. No Brasil, Senna et al. 6 estimaram a prevalência da artrite reumatoide em $0,46 \%$, o que representa quase 1 milhão de pessoas acometidas por esta doença.

A terapêutica varia de acordo com características individuais do paciente, como a tolerância à dor e a resposta a regimes prévios de tratamento, e a atividade e gravidade da doença ${ }^{3,7}$. O tratamento farmacológico é considerado o ponto chave na terapêutica da artrite reumatoide e sua implantação precoce e intensiva tem como objetivo alcançar a remissão da doença e evitar danos irreversíveis e perda da funcionalidade 8,9,10.

No Brasil, o Sistema Único de Saúde (SUS), por meio dos Componentes Básico e Especializado da Assistência Farmacêutica, disponibiliza a pacientes com artrite reumatoide medicamentos tanto para as fases iniciais de tratamento quanto para as intermediárias e avançadas. Atualmente, os medicamentos padronizados são: analgésicos, anti-inflamatórios, medicamentos modificadores do curso da doença e agentes biológicos. Em 2013, está prevista a manutenção dos agentes biológicos já dispensados (adalimumabe, etanercepte e infliximabe) e a inclusão de mais cinco representantes desta classe (golimumabe, certolizumabe pegol, rituximabe, abatacepte e tocilizumabe) 11 .

Embora a terapêutica da artrite reumatoide esteja principalmente apoiada no tratamento farmacológico, intervenções não farmacológicas, acompanhamento multiprofissional e outros procedimentos, como consultas e exames, são fundamentais para o alcance do controle da doença e melhoria da qualidade de vida do paciente 7,12,13,14.

Nos últimos anos, apesar das estratégias adotadas para ampliar o acesso a serviços de saúde e medicamentos 15, ainda existem gargalos importantes de acesso aos procedimentos necessários para o gerenciamento clínico da doença. Frequentemente, os pacientes necessitam buscar a integralidade da atenção em diferentes setores do sistema de saúde 16 .
O sistema de saúde brasileiro estrutura-se com base em três setores: o público, no qual a estrutura, os insumos e os serviços são financiados e providos pelo Estado; o privado, cujo financiamento e prestação de serviços seguem as regras do livre mercado; e o de saúde suplementar, através de planos privados de saúde, apólices de seguro ou cooperativas médicas. Os setores privados e de saúde suplementar, embora sejam regulados por agências do Estado, também não deixam de receber contribuição indireta deste, por meio de subsídios ou renúncia fiscal 16.

A utilização simultânea dos diferentes setores - público, privado e suplementar -, decorrente da incapacidade do setor público em suprir todas as demandas geradas pela artrite reumatoide, pode incrementar ainda mais o custo da doença.

Sendo assim, o presente estudo teve como objetivo estimar e caracterizar, conforme as fontes pagadoras, os custos diretos médicos relacionados ao tratamento da artrite reumatoide grave, em pacientes atendidos por meio do Componente Especializado da Assistência Farmacêutica (CEAF).

\section{Metodologia}

\section{Tipo de estudo}

Trata-se de um estudo de custo-análise 17. Os custos associados à artrite reumatoide grave foram identificados e mensurados, conforme as fontes pagadoras, por meio de um estudo observacional, descritivo e prospectivo.

\section{População e amostra}

O estudo envolveu pacientes em tratamento para a artrite reumatoide (CID-10 M05 ou M06), residentes no Município de Florianópolis, Santa Catarina, Brasil, e cadastrados no CEAF para receberem os medicamentos adalimumabe, infliximabe, etanercepte (agentes biológicos) e leflunomida (medicamentos modificadores do curso da doença). Considerando a estratégia escalonada de tratamento proposta pelo Ministério da Saúde 7 , tais pacientes foram considerados com artrite reumatoide grave.

A amostra foi constituída por todos os pacientes com idade superior a 18 anos, de ambos os sexos, e em qualquer etapa de tratamento. Foram excluídos os pacientes que não quiseram ou não puderam participar do estudo e os que realizaram apenas a entrevista inicial.

O estudo foi conduzido na Farmácia Escola, Universidade Federal de Santa Catarina (UFSC), no Município de Florianópolis. 


\section{Coleta de dados}

A coleta de dados iniciou em agosto de 2008, com um período de seis meses para captação dos pacientes. Esta ocorreu durante a dispensação dos medicamentos e foi constituída por dois momentos distintos: a entrevista inicial, realizada no primeiro mês, onde os objetivos do estudo foram expostos e os dados demográficos e socioeconômicos foram coletados; e o acompanhamento mensal e individual, durante os doze meses consecutivos, quando foram coletados os dados referentes aos recursos consumidos.

A coleta de dados foi complementada por consultas aos prontuários e aos registros de dispensação, com intuito de minimizar o viés de memória.

\section{Identificação e quantificação dos custos}

Foram considerados os custos diretos médicos associados ao tratamento da artrite reumatoide grave, às comorbidades e aos efeitos adversos decorrentes da terapêutica medicamentosa, os quais foram estimados para o período de um ano, por meio da técnica micro-costing.

Como componentes dos custos diretos médicos foram incluídos: medicamentos; administração de medicamentos parenterais; consultas médicas; consultas médicas de urgência; terapias e atividades físicas; exames complementares e hospitalizações.

A estimativa dos custos dos medicamentos incluiu aqueles utilizados para o controle da doença e de seus efeitos adversos, bem como para o tratamento das comorbidades. Os custos com administração de medicamentos parenterais foram classificados conforme as vias de administração.

Como consultas médicas foram consideradas aquelas com horário previamente agendado, enquanto como consultas médicas de urgência, aquelas realizadas em serviços de urgência e emergência, sem horário agendado.

Consideraram-se como terapias físicas: fisioterapia, pilates, acupuntura, shiatsu, watsu e yoga. Como atividades físicas foram consideradas: academia, alongamento, caminhada, exercícios físicos em casa e em áreas públicas, hidroginástica e natação.

Os exames complementares foram classificados, em: exames laboratoriais, de imagem, oftalmológicos e outros tipos. Os últimos incluem exames relacionados aos sistemas cardíaco, respiratório e circulatório.

As hospitalizações foram divididas em cirúrgicas e não cirúrgicas devido às diferenças de custo resultantes da presença e/ou ausência deste tipo de procedimento.
As fontes pagadoras utilizadas pelos pacientes para garantir o acesso aos serviços de saúde foram classificadas em: SUS e ações judiciais impetradas contra o Estado de Santa Catarina (setor público), plano de saúde (setor suplementar) e desembolso direto (setor privado).

Neste estudo, foram considerados como procedimentos: administração de medicamentos parenterais, terapias e atividades físicas, atendimento em consultas médicas de urgência ou não e exames complementares.

Os custos unitários foram estimados em Reais (R\$) e valorados, conforme as fontes pagadoras identificadas durante o estudo, utilizando-se preços de mercado, custos unitários padrões e remunerações, segundo demonstra a Tabela 1.

Calculou-se para cada representante, com exceção das hospitalizações, o produto entre as quantidades de recursos consumidos e o valor unitário de cada recurso, conforme a fonte pagadora. Em relação às hospitalizações, obtevese junto às instituições o custo total de cada hospitalização.

O custo direto médico total foi estimado pela soma dos custos de seus componentes, independente da fonte pagadora.

\section{Aspectos éticos}

O estudo foi realizado com o consentimento da Secretaria de Saúde do Estado de Santa Catarina (SES/SC) e foi aprovado pelo Comitê de Ética em Pesquisa com Seres Humanos da UFSC, sob o parecer no 103/08, conforme estabelece a Resolução no 196, do Conselho Nacional de Saúde, de 199618.

Todos os participantes foram informados e esclarecidos sobre os objetivos e finalidades do estudo, por meio do Termo de Consentimento Livre e Esclarecido, o qual foi apresentado na entrevista inicial.

\section{Resultados}

Havia 136 pacientes cadastrados no CEAF que correspondiam aos critérios de inclusão propostos. Foram excluídos 21 pacientes que não quiseram ou não puderam participar da pesquisa e três que realizaram apenas a entrevista inicial.

Durante o período de acompanhamento, ocorreram nove perdas em decorrência de: óbito $(\mathrm{n}=1)$, mudança de domicílio $(\mathrm{n}=2)$, suspensão definitiva do medicamento devido à ineficácia terapêutica $(n=1)$ e a efeitos adversos $(n=3)$ e abandono $(n=2)$. Ao final, 103 pacientes concluíram o estudo. 
Origem dos custos unitários, conforme fonte pagadora.

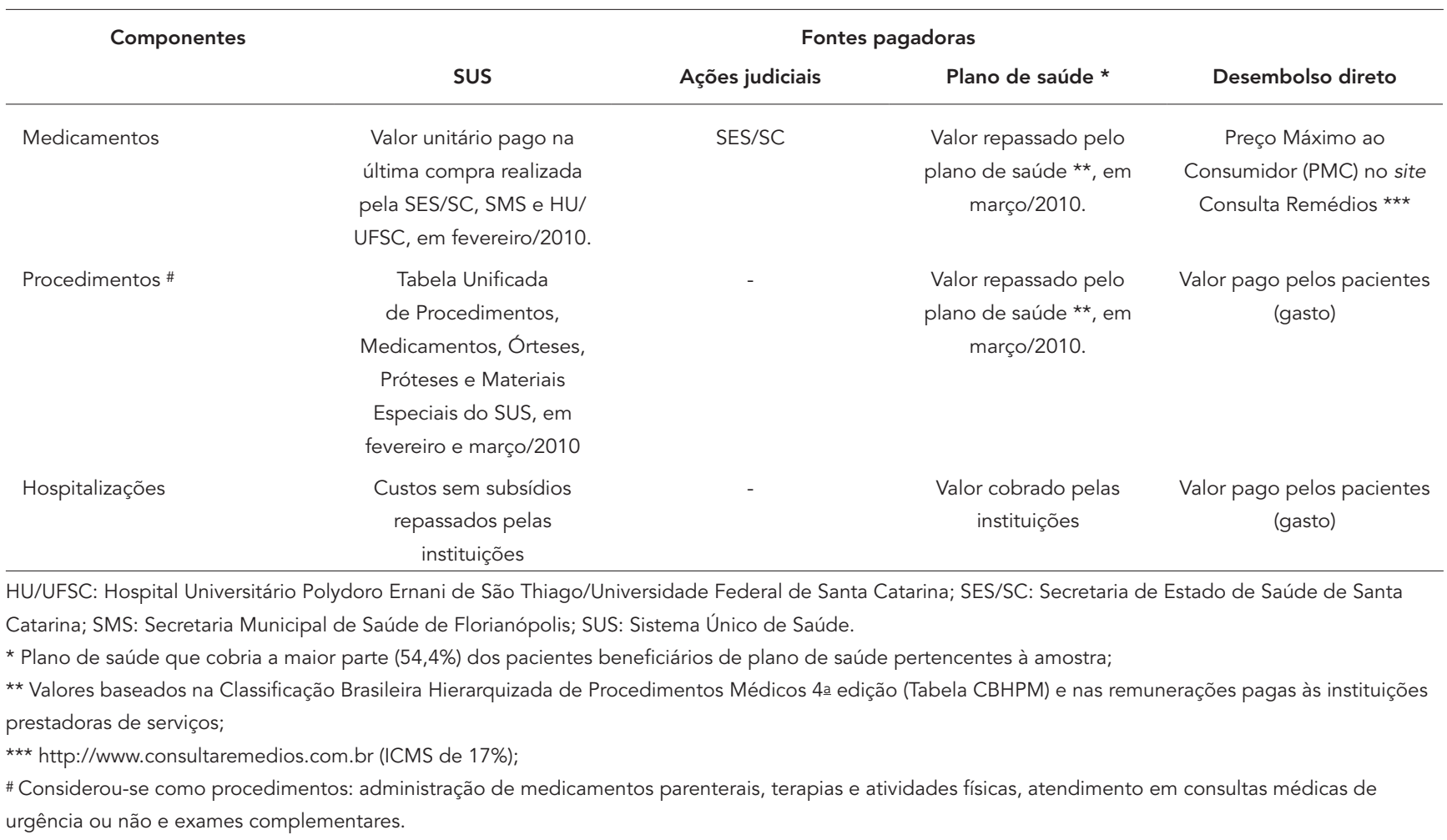

\section{Perfil da amostra}

A amostra, em sua maioria, foi constituída por pacientes do sexo feminino $(89,7 \%)$, com idade superior a 50 anos $(70,9 \%)$, escolaridade igual ou superior a 11 anos $(52,4 \%)$ e 10 anos ou mais de doença (55,3\%).

Quanto à capacidade produtiva, 14,6\% dos pacientes encontravam-se em gozo de auxílio doença previdenciário, todos em decorrência da artrite reumatoide; $43,7 \%$ eram aposentados, sendo que destes $48,9 \%$ aposentaram-se por motivo de invalidez devido à artrite reumatoide; $19,4 \%$ exerciam algum tipo de atividade remunerada; e $22,3 \%$ não exerciam atividade remunerada.

Entre os esquemas terapêuticos utilizados, predominaram as associações entre medicamentos modificadores do curso da doença e entre agentes biológicos e medicamentos modificadores do curso da doença, sendo que a associação entre leflunomida e metotrexato foi o esquema terapêutico predominante em todo o período de estudo $(41,4 \%)$. Durante o estudo, $56,3 \%$ dos pacientes permaneceram com o mesmo esquema terapêutico. Foram verificadas 58 alterações de esquemas terapêuticos, destas
$77,2 \%$ foram incorporação e abandono/suspensão de metotrexato, 10,8\% alteração entre agentes biológicos, $6,9 \%$ mudança de medicamentos modificadores do curso da doença para biológico, 3,4\% alteração entre medicamentos modificadores do curso da doença, e 1,7\% mudança de biológico para medicamentos modificadores do curso da doença. Entre os 103 pacientes acompanhados, $74,8 \%$ eram beneficiários de plano de saúde. Somente um paciente adquiriu medicamentos e realizou procedimentos exclusivamente pelo SUS, durante os 12 meses de acompanhamento.

\section{Custos diretos médicos}

O custo direto médico total, durante 12 meses de acompanhamento, referente a 103 pacientes com artrite reumatoide grave, foi de $\mathrm{R} \$ 2.045 .596,55$, o que equivale a uma média de $\mathrm{R} \$ 19.860,16$ por paciente/ano. O SUS foi a principal fonte pagadora, sendo responsável por $73,6 \%$ do custo direto médico total.

Em relação à composição do custo total, 90,8\% corresponde à aquisição de medicamentos, $2,5 \%$ às hospitalizações, $2,2 \%$ aos exames complementares e $2,1 \%$ às consultas médicas. 
Os demais componentes dos custos diretos médicos somaram $2,4 \%$ (Tabela 2 ).

O SUS foi a principal fonte pagadora dos medicamentos $(79,3 \%)$ e administrações de medicamentos parenterais $(85,4 \%)$; o plano de saúde das consultas médicas de urgência $(63,7 \%)$, exames complementares $(68,8 \%)$ e hospitalizações (85,1\%); e o desembolso direto das consultas médicas $(59,4 \%)$, atividades $(95,6 \%)$ e terapias físicas $(54,4 \%)$.

Os 103 pacientes utilizaram 1.578 medicamentos, correspondendo a uma média de $15,3 \pm 6,5$ medicamentos por paciente/ano. O número de medicamentos utilizados concomitantemente variou de dois a 16 , sendo a média de $8,6 \pm 3,3$.

O custo total com medicamentos foi de R\$ 1.857.178,99. O grupo terapêutico dos imunossupressores, o qual inclui os medicamentos modificadores do curso da doença e os agentes biológicos, foi o principal responsável por este custo, correspondendo a $86,9 \%$. Se somarmos o custo do SUS ao das ações judiciais impetradas contra o Estado de Santa Catarina, o percentual de contribuição desta fonte pagadora eleva-se para $86,4 \%$ ( $\mathrm{R} \$ 1.605 .281,03)$.

No que se refere à administração de medicamentos, 73 pacientes utilizaram medicamentos parenterais. O maior número de administrações foi realizado via subcutânea $(92,6 \%)$ e sem ônus ao paciente ou às demais fontes pagadoras, sendo na maioria das vezes administradas pelo próprio paciente, familiar ou amigo.
O SUS foi responsável por $85,4 \%$ do custo total com administrações parenterais. O maior número de administrações realizadas por intermédio do SUS ( $\mathrm{n}=1.135$ ) correspondeu aos medicamentos com administração subcutânea, porém o maior custo foi associado aos medicamentos com administração intravenosa (R\$ 15.257,26).

Foram registradas 696 consultas médicas no decorrer do estudo. O número de consultas por paciente variou entre 1-19, com média de $6,8 \pm 3,6$ consultas/paciente/ano. Entre as consultas médicas registradas, verificou-se uma média de 3,2 $\pm 1,5$ consultas especializadas em reumatologia por paciente/ano, com variação de 1-11 consultas. O número total de consultas médicas, distribuído conforme especialidade médica, fonte pagadora e custo, pode ser visualizado na Tabela 3.

O maior número de consultas médicas foi realizado por intermédio de plano de saúde $(\mathrm{n}=369)$. As consultas realizadas por desembolso direto $(n=112)$ corresponderam a $59,4 \%(R \$$ $26.030,00$ ) do custo total com este procedimento. Embora o SUS tenha representado $30,9 \%$ do número total de consultas médicas e $23,3 \%$ dos pacientes tenham consultado exclusivamente pelo SUS, esta fonte pagadora foi responsável pelo menor custo quando comparado a planos de saúde e desembolso direto.

No decorrer do estudo, quarenta pacientes realizaram consultas médicas de urgência, totalizando 72 registros. A diferença entre o número de consultas médicas de urgência procuradas por

Custo direto médico total anual em Reais (R\$), distribuído conforme seus componentes e fontes pagadoras, referente a uma amostra * de 103 pacientes em tratamento para artrite reumatoide grave.

\begin{tabular}{|c|c|c|c|c|c|c|}
\hline \multirow[t]{2}{*}{ Representante } & \multicolumn{6}{|c|}{ Fonte pagadora } \\
\hline & SUS (R\$) & $\begin{array}{c}\text { Plano de } \\
\text { saúde (R\$) }\end{array}$ & $\begin{array}{c}\text { Desembolso } \\
\text { direto (R\$) }\end{array}$ & $\begin{array}{c}\text { Ações } \\
\text { judiciais (R\$) }\end{array}$ & Total (R\$) & Total (\%) \\
\hline Medicamentos & $1.473 .636,18$ & $11.283,02$ & $240.614,94$ & $131.644,85$ & $1.857 .178,99$ & 90,8 \\
\hline Hospitalização & $5.870,29$ & $43.195,85$ & $1.676,79$ & 0,00 & $50.742,93$ & 2,5 \\
\hline Exames complementares & $8.031,30$ & $34.140,94$ & $3.802,00$ & 0,00 & $45.974,24$ & 2,2 \\
\hline Consultas médicas & $2.150,00$ & $15.638,59$ & $26.030,00$ & 0,00 & $43.818,59$ & 2,1 \\
\hline Administração de medicamentos parenterais & $15.974,83$ & $2.037,44$ & 692,56 & 0,00 & $18.704,83$ & 1,0 \\
\hline Terapias fisicas & 30,45 & $7.019,92$ & $8.400,00$ & 0,00 & $15.450,37$ & 0,7 \\
\hline Atividades físicas & 208,80 & 300,00 & $11.180,00$ & 0,00 & $11.688,80$ & 0,6 \\
\hline Consultas médicas de urgência & 374,00 & $1.297,80$ & 366,00 & 0,00 & $2.037,80$ & 0,1 \\
\hline Total & $1.506 .275,85$ & $114.913,56$ & $292.762,29$ & $131.644,85$ & $2.045 .596,55$ & 100,0 \\
\hline Total (\%) & 73,6 & 5,6 & 14,3 & 6,5 & 100,0 & \\
\hline
\end{tabular}

SUS: Sistema Único de Saúde.

* Pacientes cadastrados no Componente Especializado da Assistência Farmacêutica, na Farmácia Escola da Universidade Federal de Santa Catarina/ Prefeitura Municipal de Florianópolis. 
Número total de consultas médicas anuais, distribuídas conforme especialidades médicas, fontes pagadoras e custos, referente a uma amostra * de 103 pacientes em tratamento para artrite reumatoide grave.

\begin{tabular}{|c|c|c|c|c|c|c|c|c|}
\hline $\begin{array}{l}\text { Especialidades } \\
\text { médicas }\end{array}$ & Total (n) & SUS (n) & $\begin{array}{c}\text { Custo SUS } \\
\text { (R\$) }\end{array}$ & $\begin{array}{l}\text { Plano de } \\
\text { saúde (n) }\end{array}$ & $\begin{array}{l}\text { Custo plano } \\
\text { de saúde (R\$) }\end{array}$ & $\begin{array}{l}\text { Desembolso } \\
\text { direto }(n)\end{array}$ & $\begin{array}{c}\text { Custo } \\
\text { desembolso } \\
\text { direto (R\$) }\end{array}$ & $\begin{array}{c}\text { Custo total } \\
\text { (R\$) }\end{array}$ \\
\hline Reumatologia & 332 & 121 & $1.210,00$ & 120 & $5.728,20$ & 91 & $22.340,00$ & $29.278,20$ \\
\hline Pneumologia & 39 & 1 & 10,00 & 33 & $1.224,63$ & 5 & $1.050,00$ & $2.284,63$ \\
\hline Ortopedia & 39 & 6 & 60,00 & 29 & $1.126,19$ & 4 & 780,00 & $1.966,19$ \\
\hline Oftalmologia & 52 & 16 & 160,00 & 34 & $1.461,74$ & 2 & 300,00 & $1.921,74$ \\
\hline Cardiologia & 45 & 9 & 90,00 & 34 & $1.281,74$ & 2 & 300,00 & $1.671,74$ \\
\hline Geral & 58 & 40 & 400,00 & 17 & 630,87 & 1 & 80,00 & $1.110,87$ \\
\hline Dermatologia & 32 & 10 & 100,00 & 22 & 936,42 & 0 & 0,00 & $1.036,42$ \\
\hline Psiquiatria & 12 & 0 & 0,00 & 11 & 688,21 & 1 & 200,00 & 888,21 \\
\hline Neurologia & 7 & 0 & 0,00 & 4 & 148,44 & 3 & 680,00 & 828,44 \\
\hline Endocrinologia & 16 & 1 & 10,00 & 12 & 445,32 & 3 & 300,00 & 755,32 \\
\hline Otorrinolaringologia & 15 & 0 & 0,00 & 15 & 556,65 & 0 & 0,00 & 556,65 \\
\hline Angiologia & 15 & 3 & 30,00 & 12 & 445,32 & 0 & 0,00 & 475,32 \\
\hline Gastroenterologia & 12 & 3 & 30,00 & 9 & 333,99 & 0 & 0,00 & 363,99 \\
\hline Urologia & 7 & 0 & 0,00 & 7 & 259,77 & 0 & 0,00 & 259,77 \\
\hline Geriatria & 5 & 0 & 0,00 & 5 & 185,55 & 0 & 0,00 & 185,55 \\
\hline Ginecologia & 5 & 3 & 30,00 & 2 & 74,22 & 0 & 0,00 & 104,22 \\
\hline Infectologia & 3 & 1 & 10,00 & 2 & 74,22 & 0 & 0,00 & 84,22 \\
\hline Nefrologia & 1 & 0 & 0,00 & 1 & 37,11 & 0 & 0,00 & 37,11 \\
\hline Alergia e imunologia & 1 & 1 & 10,00 & 0 & 0,00 & 0 & 0,00 & 10,00 \\
\hline Total & 696 & 215 & $2.150,00$ & 369 & $15.638,59$ & 112 & $26.030,00$ & $43.818,59$ \\
\hline
\end{tabular}

SUS: Sistema Único de Saúde.

* Pacientes cadastrados no Componente Especializado da Assistência Farmacêutica, na Farmácia Escola da Universidade Federal de Santa Catarina/Prefeitura Municipal de Florianópolis.

meio do SUS $(n=34)$ e por meio de plano de saúde $(n=35)$ foi pequena.

A principal fonte pagadora do custo total anual com consultas médicas de urgência foi o setor de saúde suplementar (plano de saúde), correspondendo a $63,7 \%(\mathrm{R} \$ 1.297,80)$.

Entre os 103 pacientes, 20 (19,4\%) fizeram terapias físicas e o custo total foi de $\mathrm{R} \$ 15.450,37$. As terapias físicas realizadas por desembolso direto foram as que mais contribuíram com o custo total $(54,4 \%)$. O maior número de pacientes beneficiados $(\mathrm{n}=16)$ e o maior número de sessões ( $n=532$ ) ocorreram por intermédio de plano de saúde. Apenas um paciente realizou terapias físicas pelo SUS, correspondendo a um custo de $\mathrm{R} \$ 30,45$.

Nesta amostra, $31(30,1 \%)$ pacientes praticaram atividades físicas no mínimo uma vez por semana, durante três meses consecutivos. O custo total com atividades físicas foi de $\mathrm{R} \$ 11.688,80$, sendo que $95,6 \%$ foi custeado por desembolso direto, por 12 pacientes.
Foi registrada a realização de 3.642 exames complementares, durante o período de um ano. Todos os pacientes realizaram exames laboratoriais, 90 pacientes realizaram exames de imagem, sete exames oftalmológicos e 44 outros tipos de exames (por exemplo, doppler de carótidas, eletrocardiograma, ecocardiografia). Entre os exames registrados, $58,9 \%$ foram efetuados por meio de plano de saúde, $39,7 \%$ por intermédio do SUS e 1,4\% por desembolso direto. O custo total com exames complementares foi de $\mathrm{R} \$$ 45.974,24 e o setor suplementar (plano de saúde) contribuiu com $74,3 \%$ deste total, sendo também a principal fonte pagadora utilizada pelos pacientes e a responsável pelo maior número de exames realizados.

Os exames laboratoriais representaram $42,6 \%$ do custo total com exames, enquanto que os exames de imagem $37,8 \%$, os oftalmológicos $2,3 \%$ e os outros tipos $17,2 \%$. O custo médio dos exames laboratoriais foi de $\mathrm{R} \$ 6,40$ / exame. Entre os exames de imagem, o custo médio observado 
foi de $\mathrm{R} \$ 38,50$ /exame, nos exames oftalmológi$\cos \mathrm{R} \$ 48,15 /$ exame e nos outros tipos o custo foi de $\mathrm{R} \$ 73,36 /$ exame.

Durante o acompanhamento, 11 pacientes foram hospitalizados, totalizando 13 hospitalizações, sendo que em três destas foram efetuados procedimentos cirúrgicos. Entre as hospitalizações, seis ocorreram em instituições públicas por intermédio do SUS e sete em instituições privadas - seis por meio de plano de saúde e uma por desembolso direto. O custo total com hospitalizações foi de R\$50.742,93 e o plano de saúde foi responsável por $85,1 \%$ deste custo.

\section{Discussão}

O custo direto médico total estimado por paciente, em 12 meses, foi de $\mathrm{R} \$ 19.860,16$, superando o Produto Interno Bruto (PIB) per capita (R\$ 19.016,00) do ano de 201019.

A amostra analisada foi constituída, principalmente, por pacientes que utilizavam medicamentos modificadores do curso da doença, fato que diminuiu o custo médio por paciente/ano, por diluir o custo dos agentes biológicos. Isso é evidenciado pela diferença entre o custo médio anual com medicamentos dos pacientes que utilizaram medicamentos modificadores do curso da doença ( $\mathrm{R} \$ 4.424,06)$ e daqueles que fizeram uso de agentes biológicos ( $\mathrm{R} \$ 42.381,76$ ).

Os imunossupressores foram os principais responsáveis pelo custo com a aquisição de medicamentos, o que já era esperado, pois não apenas representam o grupo mais frequente, como também apresentam os maiores valores unitários. Em 2007, o Ministério da Saúde gastou cerca de $\mathrm{R} \$ 147.232 .846,74$ com a aquisição de imunossupressores para artrite reumatoide, o que correspondeu a $10,4 \%$ do gasto total do Programa de Medicamentos de Dispensação em Caráter Excepcional, antiga denominação dada ao CEAF 20.

Embora durante o estudo tenha sido constatada a ocorrência de apenas um óbito, confirmando que a morbidade relacionada à doença é superior a mortalidade, sabe-se que pacientes com artrite reumatoide têm uma sobrevida menor e apresentam taxas aumentadas de mortalidade, em comparação à população em geral $21,22,23,24$. A expectativa de vida pode cair de três a dez anos, dependendo da gravidade e da idade de início da doença 25,26. A morte ocorre em decorrência de comorbidades, as quais acometem 27 a $80 \%$ dos pacientes 23 . Dentre os fatores preditivos de mortalidade, estão idade avançada, incapacidade funcional, número de articulações acometidas, fator reumatoide positivo, nódulos reumatoides e velocidade de hemossedimentação (VHS) elevada 22,27.

Estudos envolvendo os custos associados à artrite reumatoide, realizados em diferentes países, demonstram variações entre os percentuais de participação dos componentes dos custos diretos médicos. Nos Estados Unidos, na Colômbia e na Argentina 28,29,30,31, o maior responsável pelos custos diretos médicos relacionados à doença foi a aquisição de medicamentos, tal como observado no presente estudo. Na Alemanha, as consultas com profissionais da saúde foram os principais componentes ${ }^{32}$, em contrapartida, na França, as hospitalizações foram consideradas os principais contribuintes 33 .

Considerando que a aquisição de medicamentos foi a principal responsável pelo custo direto médico total, é possível afirmar que quem paga a maior parcela da conta da artrite reumatoide, no Brasil, é o setor público, o SUS, já que este foi responsável por $79,3 \%$ do custo com a aquisição de medicamentos e por $73,6 \%$ do custo direto médico total.

Verificou-se um elevado percentual de pacientes beneficiários de planos de saúde (74,8\%). Esta informação não acompanha a tendência nacional, cujos dados referentes à Pesquisa Nacional por Amostra de Domicílios (PNAD) de 2008 demonstram que 25,9\% da população brasileira, ou seja, 49,2 milhões de pessoas dispunham de pelo menos um plano de saúde 34 . Também foi observado que a maior parte da amostra possui um bom grau de escolaridade, ao contrário do verificado em outros estudos envolvendo pacientes com artrite reumatoide no Brasil 35,36, o que pode estar relacionado ao fato de que o Estado de Santa Catarina apresenta a segunda menor taxa de analfabetismo entre pessoas com dez anos ou mais de idade do país 37 . Estas características favorecem o acesso a informações e a aquisição de produtos e serviços, como planos de saúde. Assim, é possível inferir que a amostra estudada, por utilizar simultaneamente os insumos e serviços do setor público e suplementar de saúde, tenha através deste maior facilidade de acesso aos exames complementares e às consultas médicas, o que pode contribuir para o ingresso e permanência no CEAF.

Conforme Novais \& Martins 38 , a frequência de doenças crônico-degenerativas entre beneficiários de planos de saúde é superior em relação ao SUS, o que pode estar associado a três fatores: (i) maior acesso a consultas e exames complementares (relacionados ao "risco moral" por parte dos usuários e dos prestadores de serviços), o que também facilita a detecção precoce da doença e seu monitoramento; (ii) "seleção adversa" 
dos usuários do sistema; e (iii) diferença na faixa etária entre as duas populações.

$\mathrm{Na}$ amostra estudada, observou-se que $80,6 \%$ dos pacientes não estão inseridos no mercado de trabalho e destes, 35,9\% foram afastados precocemente de suas atividades laborais em decorrência da doença. Tal fato impactaria nos custos indiretos, que não foram avaliados neste estudo, mas que aumentariam o custo total da doença. Em virtude da incapacidade laboral provocada pela artrite reumatoide, estima-se que apenas 40\% dos pacientes são capazes de trabalhar após 15 anos do diagnóstico 39. Estes achados reforçam o impacto econômico da doença, não apenas para os pacientes, seus familiares e o sistema de saúde, mas também para a sociedade.

As administrações intravenosas foram as principais responsáveis pelos custos com administrações de medicamentos, pois tratam-se de procedimentos mais complexos e requerem local, recursos humanos, materiais e monitoramento específicos para sua realização. Foi atribuído ao SUS o maior custo com administrações de medicamentos parenterais, pois, além do maior número de administrações intravenosas ter sido realizado por esta via, o valor do procedimento pelo SUS é aproximadamente três vezes superior ao valor pago pelo plano de saúde. Considera-se plausível que os medicamentos em questão, por necessitarem de condições especiais de armazenamento e de administração, tenham também o financiamento da aplicação garantido pelo SUS, pois, embora este procedimento tenha custo adicional, há garantia de efetividade do tratamento, o que se torna ainda mais importante em virtude do custo elevado dos agentes biológicos.

A média de consultas médicas realizadas pelo

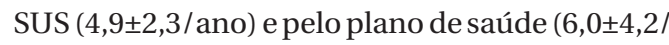
ano), durante 12 meses de acompanhamento, foi superior à verificada na PNAD de 2008 (2,5/ano e 3,7/ano, respectivamente) 38 . O maior número de consultas verificado no presente estudo pode ser atribuído à necessidade de acompanhamento periódico na atenção especializada com a reumatologia, associada à demanda por outras especialidades, em virtude das comorbidades e efeitos adversos decorrentes da terapêutica medicamentosa. Também há de se considerar que as informações utilizadas para comparação são provenientes de um inquérito de base populacional, o qual incluiu também pessoas sadias e com doenças de baixa complexidade. Além disso, a maior proporção de consultas médicas realizadas por meio de planos de saúde (48,6\% do total) pode ser condição indicativa de "risco moral", tanto por parte dos prestadores quanto dos usuários desses planos.
Entre os pacientes acompanhados, 24 (23,3\%) realizaram tanto consultas médicas por meio de plano de saúde quanto por desembolso direto. Isto pode indicar que a posse de plano de saúde não tenha sido condição suficiente para garantir $o$ acesso às consultas médicas.

As consultas realizadas por desembolso direto foram as principais responsáveis pelos custos com consultas médicas, sendo que $85,8 \%$ deste custo foram referentes a consultas com a reumatologia. Entre os 103 pacientes acompanhados, 37 realizaram consultas com esta especialidade exclusivamente por desembolso direto. Este cenário pode refletir a dificuldade de acesso à atenção especializada no SUS, o pequeno número de especialistas que atendem por intermédio de plano de saúde e/ou a alta demanda frente ao número limitado de especialistas e/ou de consultas ofertadas por eles.

A média de consultas médicas realizadas com a reumatologia por meio do SUS foi superior a de consultas por desembolso direto. Isso pode sugerir que o paciente que consegue acesso ao especialista pelo SUS é atendido com maior frequência, o que reflete que há um gargalo no acesso ao setor público e não na continuidade do acompanhamento. Este fato também pode estar associado ao elevado valor da consulta por desembolso direto, o que limita o acompanhamento periódico.

As terapias físicas proporcionam aumento e manutenção da mobilidade articular e contribuem para a autonomia do paciente na realização de suas atividades de vida diária 14,40,41. Entretanto, se observou que apenas vinte pacientes as realizaram, destes dez frequentaram-nas continuamente por no mínimo três meses; e os demais utilizaram-nas em virtude de problemas musculoesqueléticos agudos e traumas. Entre os vinte pacientes, 17 realizaram por intermédio de plano de saúde e/ou por desembolso direto, o que pode sugerir que a oferta de terapias físicas pelo SUS ainda é escassa, fato que limita sua utilização aos indivíduos economicamente privilegiados ou aos que possuem planos de saúde.

Além da terapêutica física e farmacológica, evidências científicas têm demonstrado, ao longo dos anos, os benefícios da atividade física no tratamento da artrite reumatoide em ambiente clínico, bem como em ambiente doméstico, com supervisão profissional $42,43,44$. Apenas 31 pacientes informaram realizar atividades físicas regularmente. Os demais, quando questionados sobre a prática de atividades físicas, demonstravam-se surpresos e afirmavam a impossibilidade de realizá-las em virtude da doença ou de proibição médica. Diante do perfil de terapias e atividades físicas, verifica-se que o plano terapêutico 
dos pacientes pertencentes à amostra é, basicamente, centrado na terapêutica medicamentosa.

Os exames laboratoriais, independente da fonte pagadora, foram os mais realizados. Isto porque estes são solicitados em média em intervalos trimestrais e são mais baratos e mais acessíveis, além de fornecerem informações relevantes sobre a atividades da doença e o quadro do paciente. Os exames de imagem apresentam maior custo e, comumente, são solicitados semestralmente ou anualmente como monitoramento, ou em algumas situações específicas, tais como: traumas, investigação de alguma queixa ou anormalidade.

Assim como as consultas médicas, a média de exames realizados por beneficiários de plano de saúde foi superior a dos usuários do SUS, o que pode estar associado à facilidade de acesso por parte dos beneficiários de plano de saúde e a dificuldades de acesso e falhas de gerenciamento enfrentadas pela população usuária do sistema público.

Embora o número de hospitalizações custeadas por intermédio do SUS e por plano de saúde seja semelhante, este último foi responsável por $85,1 \%$ do custo total com hospitalizações. Entre as hospitalizações registradas, destaca-se o caso de uma hospitalização custeada pelo SUS, cuja justificativa foi a necessidade de realização de exame de alta complexidade. Trata-se de caso-índice que aponta falha na oferta de procedimentos de média e alta complexidade em nível ambulatorial e no gerenciamento do sistema. Esta hospitalização custou R $\$ 1.922,88$, enquanto o exame necessário custaria $\mathrm{R} \$ 138,63$.

Em virtude do critério de inclusão proposto - cadastro no CEAF para receber os agentes biológicos e leflunomida - a amostra foi constituída por pacientes com artrite reumatoide grave, que apresentavam deformidades importantes, comorbidades, comprometimento sistêmico e reatores de fase aguda elevados. Como a intensidade da utilização de recursos está fortemente relacionada às condições clínicas dos pacientes, tais características condicionam os custos com a doença. O critério de inclusão também pode ter influenciado no fato de o SUS ser considerado a principal fonte pagadora do tratamento da artrite reumatoide, já que os pacientes para serem incluídos na amostra deveriam estar cadastrados no CEAF. Também não foram incluídos alguns componentes dos custos diretos médicos tais como materiais auxiliares (órteses e próteses) e salários dos profissionais; custos diretos não médicos como adaptações da residência, bem como custos indiretos (benefícios da Previdência Social), custos de overhead (manutenção de equipamentos e dependências físicas) e os custos intangíveis, sugerindo que o custo relacionado à doença possa ser superior ao encontrado.

Estudos de custo-análise ocupam papel central em todas as avaliações econômicas, mas recebem relativamente pouca atenção pelos analistas por entenderem que são estudos deficientes na especificação da perspectiva do estudo, na estimação das quantidades e dos preços e mesmo na identificação dos anos aos quais os custos se aplicam 17. Cientes das restrições metodológicas, os autores foram extremamente cuidadosos ao descrever os métodos de estimação das quantidades e preços utilizados nos cálculos dos custos. E enfim, como uma "externalidade" dos resultados foi possível traçar um claro perfil de como uma população de indivíduos com uma doença crônico-degenerativa altamente demandante de recursos de saúde transita pela composição público-privada que caracteriza o sistema de saúde brasileiro. 


\section{Resumen}

El tratamiento de la artritis reumatoide incluye la utilización de medicamentos, terapias no farmacológicas, consultas médicas, exámenes clínicos, entre otros procedimientos. El artículo presenta, de acuerdo con las fuentes pagadoras, los costes médicos directos de tratamiento de artritis reumatoide. Se trata de un estudio de análisis de costes que incluyó 103 pacientes con artritis reumatoide severa, atendidos a través del Dispositivo Especializado de Asistencia Farmacéutica en Florianópolis, Santa Catarina, Brasil. El coste médico

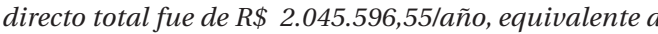
$R \$ 19.860,16$ por pacientelaño. Del coste total, un 90,8\% correspondió a medicamentos; $2,5 \%$ a hospitalizaciones y un 2,2\% a exámenes clínicos. El sector público fue responsable de un $73,6 \%$ del coste médico directo total y de un 79,3\% del coste de adquisición de medicamentos. El análisis de costes permitió trazar un perfil de cómo una población portadora de una enfermedad crónicodegenerativa, con una alta demanda de recursos transita por el sistema público-privado que caracteriza al sistema de salud brasileño.

Artritis Reumatoide; Costos de la Atención en Salud; Evaluación de Tecnologías de Salud

\section{Colaboradores}

F. B. Buendgens participou da concepção do estudo, coleta e análise de dados e redação do artigo. C. R. Blatt, S. N. Leite e A. C. E. Marasciulo contribuíram com revisão crítica e aprovação final da versão a ser publicada. M. R. Farias participou da concepção do estudo, revisão crítica e aprovação final da versão a ser publicada.

\section{Agradecimentos}

Os autores agradecem aos gestores e funcionários da Farmácia Escola da UFSC/PMF, da Secretaria de Estado da Saúde de Santa Catarina e da Secretaria Municipal de Saúde de Florianópolis por viabilizarem a estrutura física e o suporte necessário para a realização deste trabalho e aos pacientes incluídos neste estudo, pois apesar da condição fragilizada, não mediram esforços para colaborar com a realização do mesmo.

\section{Conflito de interesses}

Não declarado.

\section{Referências}

1. Pinto MRC, Miguel RCC, Rezende GG. Rheumatoid arthritis treatment. Rev Bras Reumatol 2006; 46:219-23.

2. Venables PJW, Maini RN. Clinical features of rheumatoid arthritis. UpToDate 2008; 16.1. http:// www.uptodate.com/contents/clinical-features-ofrheumatoid-arthritis?source $=$ see link.

3. Harris Jr. ED. Clinical features of rheumatoid arthritis. In: Harris ED, Budd R, Genovese M, Firestein G, Sargent J, Sledge C, editors. Kelley's textbook of rheumatology. $7^{\text {th }}$ Ed. Philadelphia: ElsevierSaunders; 2005. p. 1043-100.

4. Scott DL, Wolfe F, Huizinga TW. Rheumatoid arthritis. Lancet 2010; 376:1094-108.

5. World Health Organization. Chronic diseases and health promotion. http://www.who.int/chp/top ics/rheumatic/en/ (acessado em 21/Dez/2012).

6. Senna ER, De Barros AL, Silva EO, Costa IF, Pereira LV, Ciconelli RM, et al. Prevalence of rheumatic diseases in Brazil: a study using the COPCORD approach. J Rheumatol 2004; 31:594-7.

7. Ministério da Saúde. Portaria SCTIE no 66, de 6 de novembro de 2006. Aprova o Protocolo Clínico e Diretrizes Terapêuticas - artrite reumatoide. Diário Oficial da União 2006; 7 nov.

8. Mottonen T, Hannonen P, Korpela M, Nissilä M, Kautiainen H, Ilonen J, et al. Delay to institution of therapy and induction of remission using singledrug or combination-disease-modifying antirheumatic drug therapy in early rheumatoid arthritis. Arthritis Rheum 2002; 46:894-8.
9. Breedveld FC, Kalden JR. Appropriate and effective management of rheumatoid arthritis. Ann Rheum Dis 2004; 63:627-33.

10. Haroon N, Misra R, Aggarwal A. Tailor-made therapy in rheumatoid arthritis: fact or fiction? Isr Med Assoc J 2008; 10:139-41.

11. Ministério da Saúde. Portaria no 24, 10 de setembro de 2012. Torna pública a decisão de incorporar os medicamentos golimumabe, certolizumabe pegol, rituximabe, abatacepte e tocilizumabe, bem como a manutenção dos medicamentos infliximabe, adalimumabe e etanercepte para o tratamento da artrite reumatoide no Sistema Único de Saúde (SUS). Diário Oficial da União 2012; 11 set.

12. Blom M, Van Riel PL. Management of established rheumatoid arthritis with an emphasis on pharmacotherapy. Best Pract Res Clin Rheumatol 2007; 21:43-57.

13. Combe B. Early rheumatoid arthritis: strategies for prevention and management. Best Pract Res Clin Rheumatol 2007; 21:27-42.

14. Smolen J, Aletaha D. The burden of rheumatoid arthritis and acess to treatment: a medical overview. Eur J Health Econ 2008; 8 Suppl 2:S39-47.

15. Araújo ALA, Pereira LRL, Ueta JM, Freitas O. Perfil da assistência farmacêutica na atenção primária do Sistema Único de Saúde. Ciênc Saúde Coletiva 2008; 13 Suppl:S611-7. 
16. Paim J, Travassos C, Almeida C, Bahia L, Macinko J. O sistema de saúde brasileiro: história, avanços e desafios. Lancet 2011; 377:1778-97.

17. Drummond MF, Schulper MJ, Torrance GW, O’Brien BJ, Stoddart GL. Methods for the economic evaluation of heath care programmes. 3rd Ed. London: Oxford Medical Publications; 2005.

18. Conselho Nacional de Saúde. Resolução no 196 de 10 de outubro de 1996. Estabelece os requisitos para realização de pesquisa clínica de produtos para saúde utilizando seres humanos. Diário Oficial da União 1996; 16 out.

19. Instituto Brasileiro de Geografia e Estatística. Em 2010, PIB varia 7,5\% e fica em $\mathrm{R} \$ 3,675$ trilhões. http://saladeimprensa.ibge.gov.br/noticias?view= noticia\&id=1\&busca=1\&idnoticia=1830 (acessado em 22/Jan/2012).

20. Carias CM, Vieira FS, Giordano CV, Zucchi P. Medicamentos de dispensação excepcional: histórico e gastos do Ministério da Saúde do Brasil. Rev Saúde Pública 2011; 45:233-40.

21. Alamanos Y, Drosos AA. Epidemiology of adult rheumatoid arthritis. Autoimmun Rev 2005; 4:130-6.

22. Gabriel SE. Why do people with rheumatoid arthritis still die prematurely? Ann Rheum Dis 2008; 67 Suppl 3:iii30-4.

23. Smolen J, Aletaha D. The burden of rheumatoid arthritis and acess to treatment: a medical overview. Eur J Health Econ 2008; 8 Suppl 2:S39-47.

24. Kirchhoff T, Ruof J, Mittendorf T, Rihl M, Bernateck M, Mau W, et al. Cost of illness in rheumatoid arthritis in Germany in 1997-98 and 2002: cost drivers and cost savings. Rheumatology (Oxford) 2011; 50:756-61.

25. Turesson C, O'Fallon WM, Crowson CS, Gabriel SE, Matteson EL. Occurrence of extra-articular disease manifestations is associated with excess mortality in a community based cohort of patients with rheumatoid arthritis. J Rheumatol 2002; 29:62-7.

26. Brenol CV, Monticielo OA, Xavier RM, Brenol JCT. Artrite reumatóide e aterosclerose. AMB Rev Assoc Med Bras 2007; 53:465-70.

27. Pereira IA. Artrite reumatóide. ACM Arq Catarin Med 2007; 36:95-101.

28. Audisio MJ, Strusberg I, Orellana Barrera SD, Drenkard CM, Barberis G, Gamrón S, et al. Costo directo semestral por artritis reumatoidea en pacientes que concurren a un hospital universitario. Rev Fac Cien Med Univ Nac Cordoba 2003; 60: 35-41.

29. Michaud K, Messer J, Choi HK, Wolfe F. Direct medical costs and their predictors in patients with rheumatoid arthritis: a three-year study of 7,527 patients. Arthritis Rheum 2003; 48:2750-62.

30. Kavanaugh A. The pharmacoeconomics of newer therapeutics for rheumatic diseases. Rheum Dis Clin North Am 2006; 32:45-56.

31. Mora C, González A, Díaz J, Quintana G. Costos directos de la artritis reumatoide temprana en el primer año de atención:simulación de tres situaciones clínicas en un hospital universitario de tercer nivel em Colombia. Biomédica 2009; 29:43-50.
32. Verstappen A, Verkleij H, Bijlsma J, Buskens E, Kruize A, Heurkens A, et al. Determinants of direct costs in Dutch rheumatoid arthritis patients. Ann Rheum Dis 2004; 63:817-24.

33. Guillemin F, Durieux S, Daurès JP, Lafuma A, Saraux A, Sibilia J, et al. Costs of rheumatoid arthritis in France: a multicenter study of 1109 patients managed by hospital-based rheumatologists. J Rheumatol 2004; 31:1297-304.

34. Instituto Brasileiro de Geografia e Estatística. Pesquisa Nacional por Amostra de Domicílio: um panorama da saúde no Brasil. Acesso e utilização dos serviços, condições de saúde e fatores de risco e proteção à saúde, 2008. Rio de Janeiro: Instituto Brasileiro de Geografia e Estatística; 2010.

35. Chermont GC, Kowalski SC, Ciconelli RM, Ferraz MB. Resource utilization and the cost of rheumatoid arthritis in Brazil. Clin Exp Rheumatol 2008; 26:24-31.

36. Azevedo ABC, Ciconelli RM, Ferraz MB. Indirect costs of rheumatoid arthritis in Brazil. Value Health 2008; 11:869-77.

37. Instituto Brasileiro de Geografia e Estatística. Censo Demográfico 2010: características da população e dos domicílios. Resultados do universo. Rio de Janeiro: Instituto Brasileiro de Geografia e Estatística; 2011.

38. Novais M, Martins CB. Perfil dos beneficiários de planos e SUS e o acesso a serviços de saúde - PNAD 2003 e 2008. http://www.iess.org.br/ TDIESS00352010PNAD003_2008.pdf (acessado em 03/Jan/2013).

39. Kvien TK. Epidemiology and burden of illness of rheumatoid arthritis. Pharmacoeconomics 2004; 22(2 Suppl 1):S1-12.

40. Gossec L, Pavy S, Pham T, Constantin A, Poiraudeau $\mathrm{S}$, Combe $\mathrm{B}$, et al. Non-pharmacological treatments in early rheumatoid arthritis: clinical practice guidelines based on published evidence and expert opinion. Joint Bone Spine 2006; 73:396402.

41. Mota LMH, Cruz BA, Brenol CV, Pereira IA, Rezende-Fronza LS, Bértolo MB, et al. Consenso 2012 da Sociedade Brasileira de Reumatologia para o tratamento da artrite reumatoide. Rev Bras Reumatol 2012; 52:135-74.

42. Jong Z, Vliet Vlieland TPM. Safety of exercise in patients with rheumatoid arthritis. Curr Opin Rheumatol 2005; 17:177-82.

43. Gaudin P, Leguen-Guegan S, Allenet B, Baillet A, Grange L, Juvin R. Is dynamic exercise beneficial in patients with rheumatoid arthritis? Joint Bone Spine 2008; 75:11-7.

44. Metsios GS, Stavropoulos-Kalinoglou A, Sandoo A, van Zanten JJ, Toms TE, John H, et al. Vascular function and inflammation in rheumatoid arthritis: The role of physical activity. Open Cardiovasc Med J 2010; 4:89-96.

Recebido em 28/Jan/2013

Versão final reapresentado em 18/Jul/2013

Aprovado em 19/Jul/2013 